\title{
Boltzmann Equation Analysis of Electron Transport in a $\mathrm{N}_{2}-\mathrm{O}_{2}$ Streamer Discharge
}

\author{
Saša Dujko ${ }^{1,2,3 *}$, Ute Ebert ${ }^{1}$, Ronald D. White ${ }^{3}$, and Zoran Lj. Petrović ${ }^{2}$ \\ ${ }^{1}$ Centrum voor Wiskunde en Informatica (CWI), P. O. Box 94079, 1090 GB Amsterdam, The Netherlands \\ ${ }^{2}$ Institute of Physics, University of Belgrade, P. O. Box 68, Zemun, Belgrade 11080, Serbia \\ ${ }^{3}$ ARC Centre for Antimatter-Matter Studies, School of Engineering and Physical Science, James Cook University, \\ Townsville, Queensland 4810, Australia
}

Received November 23, 2010; accepted March 28, 2011; published online August 22, 2011

\begin{abstract}
A comprehensive investigation of electron transport in $\mathrm{N}_{2}-\mathrm{O}_{2}$ mixtures has been carried out using a multi term theory for solving the Boltzmann equation instead of conventional two term theory often employed in plasma modeling community. We focus on the way in which the transport coefficients and spatially resolved transport data are influenced by the amount of $\mathrm{O}_{2}$ in the mixture. Emphasis is placed upon the explicit and implicit effects of non-conservative collisions, ionization and attachment on various transport coefficients. In particular, the effects of three-body attachment for electrons on various transport data are considered. It is found that the differences between two sets of transport coefficients, bulk and flux, resulting from the explicit influence of non-conservative collisions is sensitive to the quantity of $\mathrm{O}_{2}$ in the mixture.

(C) 2011 The Japan Society of Applied Physics
\end{abstract}

\section{Introduction}

Streamers are growing filaments of weakly-ionized nonstationary plasma produced by an ionization front that moves through non-ionized matter. ${ }^{1)}$ They have applications in diverse areas of science and technology ranging from their role in creating lighting and transient luminous events in the upper atmosphere ${ }^{2)}$ to industrial applications such as the treatment of polluted gases and water ${ }^{3)}$ and plasma enhanced vapor deposition in microelectronics. ${ }^{4)}$ There have been numerous simulations of streamers (see, e.g., ref. 1 and references therein), but the recent three-dimensional selfconsistent hybrid model of $\mathrm{Li}$ et al. ${ }^{5)}$ is of special interest. In this model, the fast non-equilibrium electrons in the leading part of the ionization front are treated by a Monte Carlo simulation while the low-energy electrons in the rest of the domain are treated using a fluid model. The fluid part is based on the local field approximation and requires the tabulation of electron transport coefficients as a function of the reduced electric field. The fluid model is coupled with the Monte Carlo model via a model interface. To ensure the stable and correct interaction between these two models, the correct implementation of swarm transport data and the accuracy of their calculations are critical steps in modeling. Moreover, in this work we highlight aspects of swarm transport theory and transport coefficient data that are important for further improvement of both fluid and hybrid models of streamers. This is the main avenue we explore in this work.

In this paper we solve the Boltzmann equation for electron swarms undergoing ionization and attachment in mixtures of molecular $\mathrm{N}_{2}$ and $\mathrm{O}_{2}$. Values and general trends in the profiles of the mean energy, rate coefficients, drift velocity and diffusion tensor are reported here. The investigation has resulted in a database of transport data which is applicable for a wide range of potential applications, although we focused upon the provision and correct implementation of swarm data within fluid modeling of a streamer discharge. Another important issue considered in this work is the explicit and implicit effects of three-body attachment on electron transport. Electron attachment in molecular oxygen

*Present address: Centrum voor Wiskunde en Informatica, Amsterdam, The Netherlands. E-mail address: S.Dujko@cwi.nl is an important process with a variety of applications ranging from ozone production to the analysis of the streamers and other types of atmospheric plasmas. This process has usually been treated through two-body dissociative attachment, and so far only a limited number of theoretical investigations from the kinetic theory point of view into the three-body, pressure dependent attachment process for electrons has been published. ${ }^{6-8)}$ We feel that a rigorous analysis of the three-body attachment process in the context of modern kinetic theory is long overdue, and the present paper takes the first steps in this direction, particularly for streamer modeling. ${ }^{9-12)}$ In $\S 2$, we discuss the hydrodynamic regime and identify the difference in the bulk and flux transport coefficients. A brief description of the theoretical multi term solution of the Boltzmann equation is presented for conditions where non-conservative collisions (attachment and/or ionization) are operative. In $\S 3$, we present the results of a systematic study of electron transport in the mixtures of molecular $\mathrm{N}_{2}$ and $\mathrm{O}_{2}$. Transport coefficients are presented for various $\mathrm{N}_{2}-\mathrm{O}_{2}$ mixtures as a function of the reduced electric field $E / n_{0}$ (where $n_{0}$ is the gas number density).

\section{Theory}

The behavior of charged particle swarms in gases under the influence of electric and magnetic fields is described by the phase-space distribution function $f(\boldsymbol{r}, \boldsymbol{c}, t)$ representing the solution of the Boltzmann equation

$$
\frac{\partial f}{\partial t}+\boldsymbol{c} \cdot \frac{\partial f}{\partial \boldsymbol{r}}+\frac{e \boldsymbol{E}}{m} \cdot \frac{\partial f}{\partial \boldsymbol{c}}=-J\left(f, f_{0}\right)
$$

where $\boldsymbol{r}$ and $\boldsymbol{c}$ denote the position and velocity co-ordinates, $e$ and $m$ are the charge and mass of the electrons and $t$ is time. The electric field is assumed spatially homogeneous with magnitude $E$. Swarm conditions are assumed to apply and $J\left(f, f_{0}\right)$ denotes the rate of change of $f$ due to binary collisions with the neutral molecules only. The original Boltzmann collision operator ${ }^{13)}$ and its semiclassical generalization $^{14)}$ are used for elastic and inelastic processes respectively. The attachment and ionization collision operators employed are detailed by Ness and Robson. ${ }^{15)}$

\subsection{Hydrodynamic regime and definition of transport coefficients}

Experimental investigations of electron swarm behavior are 
generally made by sampling charged particle currents or charged particle densities:

$$
n(\boldsymbol{r}, t)=\int f(\boldsymbol{r}, \boldsymbol{c}, t) d \boldsymbol{c}
$$

The connection between experiment and theory is made through the equation of continuity

$$
\frac{\partial n(\boldsymbol{r}, t)}{\partial t}+\nabla \cdot \boldsymbol{\Gamma}=S(\boldsymbol{r}, t)
$$

where $\boldsymbol{\Gamma}=n\langle\boldsymbol{c}\rangle$ is the particle flux and $S(\boldsymbol{r}, t)$ represents the production rate per unit volume per unit time arising from nonconservative collisional processes.

Far from boundaries, sources and sinks, the hydrodynamic regime is assumed to apply. In this regime, the spacetime dependence of the phase space distribution function is expressible in terms of linear functionals of $n(\boldsymbol{r}, t)$. A sufficient functional relationship between $f(\boldsymbol{r}, \boldsymbol{c}, t)$ (and associated velocity moments) and $n(\boldsymbol{r}, t)$ in the case of weak gradients is the well-known expansion

$$
f(\boldsymbol{r}, \boldsymbol{c}, t)=\sum_{k=0}^{\infty} f^{k}(\boldsymbol{c}) \odot(-\nabla)^{k} n(\boldsymbol{r}, t),
$$

where $f^{k}(\boldsymbol{c})$ are tensors of rank $k$ and $\odot$ denotes a $k$-fold scalar product.

Assuming the functional relationship (4) the flux $\Gamma$ and source term $S(\boldsymbol{r}, t)$ in (3) are expanded as

$$
\begin{aligned}
& \boldsymbol{\Gamma}=\boldsymbol{W}^{(\star)} n(\boldsymbol{r}, t)-\boldsymbol{D}^{(\star)} \cdot \nabla n(\boldsymbol{r}, t), \\
& S(\boldsymbol{r}, t)=S^{(0)} n(\boldsymbol{r}, t)-\boldsymbol{S}^{(1)} \cdot \nabla n(\boldsymbol{r}, t)+\boldsymbol{S}^{(2)}: \nabla \nabla n(\boldsymbol{r}, t),
\end{aligned}
$$

where $\boldsymbol{W}^{(\star)}$ and $\boldsymbol{D}^{(\star)}$ define, respectively, the flux drift velocity and $f u x$ diffusion tensor. Substitution of expansion (5) and (6) into the continuity equation (3) yields the diffusion equation

$$
\frac{\partial n}{\partial t}+\boldsymbol{W} \cdot \nabla n-\boldsymbol{D}: \nabla \nabla n=-R_{a} n,
$$

which define the bulk transport coefficients

$$
\begin{aligned}
& R_{a}=-S^{(0)} \quad \text { (reaction rate), } \\
& \boldsymbol{W}=\boldsymbol{W}^{(\star)}+\boldsymbol{S}^{(1)} \quad \text { (bulk drift velocity), } \\
& \boldsymbol{D}=\boldsymbol{D}^{(\star)}-\boldsymbol{S}^{(2)} \quad \text { (bulk diffusion tensor). }
\end{aligned}
$$

The basic difference between the bulk and flux transport coefficients should now be apparent. The bulk drift velocity is displacement of the mean position of the electron swarm and it characterizes the motion of the total ensemble of electrons. The presence of the electric field results in a spatial variation in the energy throughout the swarm. Under such conditions, the presence of non-conservative collisions (ionization/ attachment) may lead to a change in the position of the center-of-mass of the swarm. This effect on the bulk drift velocity is denoted by $S^{(1)}$. On the other hand, the flux drift velocity $W^{(\star)}$ represents the rate of change of the position of the center-of-mass due to the electric field only and can be interpreted as the mean velocity of the electrons. Likewise the flux diffusion tensor $D^{(\star)}$ represents the rate of spreading of the swarm due to $\boldsymbol{E}$ and $\nabla n$. The presence of non-conservative collisions may result in the variation of $\nabla n$ throughout the swarm and a subsequent variation in the rate of change of the mean squared width of the swarm. Such effects are expressed by the second rank tensor $S^{(2)}$. Obviously, in the absence of non-conservative collisions the $S^{(k)}$ source/sink terms describing the production/loss of electrons vanish and the bulk and flux transport coefficients coincide.

\subsection{Spherical harmonic decomposition of Boltzmann equation}

The theoretical foundations for the solution of eq. (1) under non-conservative conditions are formulated by Robson and Ness. ${ }^{15,16)}$ In 1993 Ness $^{17)}$ extended the theory and general formalism for non-conservative charged particle swarms under the influence of electric and magnetic fields crossed at arbitrary angle. Dujko et al. ${ }^{18)}$ made a further extension of the theory and the associated computer code into the domain of time dependent electric and magnetic fields crossed at arbitrary angle. Although the methods and techniques are by now standard and the reader is referred to recent reviews ${ }^{19,20)}$ for details, in what follows we briefly review the spherical harmonic decomposition of the Boltzmann equation.

In brief, the hierarchy of kinetic equations is derived through a series of three expansions:

(a) The angular component of the velocity dependence of $f(\boldsymbol{r}, \boldsymbol{c}, t)$ is represented in terms of an expansion in spherical harmonics:

$$
f(\boldsymbol{r}, \boldsymbol{c}, t)=\sum_{l=0}^{\infty} \sum_{m=0}^{\infty} f_{m}^{(l)}(\boldsymbol{r}, c, t) Y_{m}^{[l]}(\hat{\boldsymbol{c}}),
$$

where $Y_{m}^{[l]}(\hat{\boldsymbol{c}})$ are spherical harmonics and $\hat{\boldsymbol{c}}$ denotes the angles of $\boldsymbol{c}$.

(b) In the hydrodynamic regime, the spatial dependence of $f(\boldsymbol{r}, \boldsymbol{c}, t)$ is represented by

$$
f(\boldsymbol{r}, \boldsymbol{c}, t)=\sum_{s=0}^{2} \sum_{\lambda=0}^{s} f(\operatorname{lm} \mid s \lambda ; c) G_{m}^{(s \lambda)} n(\boldsymbol{r}, t),
$$

where $G_{m}^{(s \lambda)}$ is the irreducible gradient operator.

(c) The speed distribution function is represented in terms of modified Sonine polynomials about a Maxwellian at a temperature $T_{\mathrm{b}}$ :

$$
f(\operatorname{lm} \mid s \lambda ; c)=\omega(\alpha, c) \sum_{\nu=0}^{\infty} F(\nu \operatorname{lm} \mid s \lambda ; \alpha) R_{\nu l}(\alpha c),
$$

where

$$
\begin{aligned}
\omega(\alpha, c) & =\left(\frac{\alpha^{2}}{2 \pi}\right)^{3 / 2} \exp \left(\frac{-\alpha^{2} c^{2}}{2}\right) \\
\alpha^{2} & =\frac{m}{k T_{\mathrm{b}}}, \\
R_{\nu l}(\alpha c) & =N_{\nu l}\left(\frac{\alpha c}{\sqrt{2}}\right)^{l} S_{l+1 / 2}^{(v)}\left(\frac{\alpha^{2} c^{2}}{2}\right), \\
N_{\nu l}^{2} & =\frac{2 \pi^{2 / 3} v !}{\Gamma(v+l+3 / 2)},
\end{aligned}
$$

and $S_{l+1 / 2}^{(\nu)}\left(\alpha^{2} c^{2} / 2\right)$ are Sonine polynomials.

Using the orthonormality conditions of the spherical harmonics and modified Sonine polynomials, the following hierarchy of kinetic equations follows:

$$
\begin{aligned}
& \sum_{v^{\prime}=0}^{\infty} \sum_{l^{\prime}=0}^{\infty}\left[\partial_{t} \delta_{v v^{\prime}} \delta_{l l^{\prime}}+n_{0} J_{v v^{\prime}}^{l}(\alpha) \delta_{l l^{\prime}}-R_{a} \delta_{v v^{\prime}} \delta_{l l^{\prime}}\right. \\
& \quad+i a \alpha\left(l^{\prime} m 10 \mid l m\right)\left\langle v l \| K^{[1]}|| v^{\prime} l^{\prime}\right\rangle \\
& \left.\quad-n_{0} J_{0 v^{\prime}}^{0}(\alpha) F(v l 0 \mid 00 ; \alpha)\left(1-\delta_{s 0} \delta_{\lambda 0}\right) \delta_{l^{\prime} 0} \delta_{m 0}\right] F\left(v^{\prime} \operatorname{lm} \mid s \lambda ; \alpha\right) \\
& \quad=X(v \operatorname{lm} \mid s \lambda ; \alpha)
\end{aligned}
$$


where $R_{a}$ is the reaction rate. The reduced matrix elements of the collision operator $J_{v v^{\prime}}^{l}(\alpha)$ and velocity derivative $\left\langle\nu l|| K^{[1]} \| v^{\prime} l^{\prime}\right\rangle$ are defined by eqs. (11) and (12) given in ref. 15 while $\left(l^{\prime} m 10 \mid l m\right)$ is a Clebsch-Gordan coefficient. The explicit expression for the RHS are given in refs. 15 and 18.

Equation (18) is solved by truncation of the $v$ and $l$ indices to $v_{\max }$ and $l_{\max }$, respectively. These values are independently increased until the desired convergence is obtained. $T_{\mathrm{b}}$ is not equal to the neutral gas temperature (the twotemperature method) and is used as a free parameter to optimize the convergence. After truncation, we have a hierarchy of coupled complex equations. This sparse system of equations is solved using standard sparse inversion routines.

\subsection{Transport properties}

The transport coefficients of interest [attachment rate $k_{\mathrm{a}}$; bulk drift velocity $W$; longitudinal $\left(D_{\mathrm{L}}\right)$ and transverse $\left(D_{\mathrm{T}}\right)$ diffusion coefficients] are related to the calculated moments via

$$
\begin{aligned}
k_{\mathrm{a}}= & n_{0} \sum_{v^{\prime}=0}^{\infty} J_{0 v^{\prime}}^{0}(\alpha) F\left(v^{\prime} 00 \mid 00\right), \\
W= & \frac{i}{\alpha} F(010 \mid 00)-i n_{0} \sum_{v^{\prime}=1}^{\infty} J_{0 v^{\prime}}^{0}(\alpha) F\left(v^{\prime} 00 \mid 11\right), \\
D_{\mathrm{L}}= & -\frac{1}{\alpha} F(010 \mid 11)-n_{0} \sum_{v^{\prime}=0}^{\infty} J_{0 v^{\prime}}^{0}\left[F\left(v^{\prime} 00 \mid 20\right)\right. \\
& \left.-\sqrt{2} F\left(v^{\prime} 00 \mid 22\right)\right], \\
D_{\mathrm{T}}= & -\frac{1}{\alpha} F(011 \mid 11) \\
& -n_{0} \sum_{v^{\prime}=0}^{\infty} J_{0 v^{\prime}}^{0}\left[F\left(v^{\prime} 00 \mid 20\right)+\frac{1}{\sqrt{2}} F\left(v^{\prime} 00 \mid 22\right)\right],
\end{aligned}
$$

where the components involving summations constitute the explicit non-conservative effects on the transport coefficients, while the remainders constitute the flux contribution. The spatially homogeneous mean energy is given by

$$
\varepsilon=\frac{3}{2} k T_{\mathrm{b}}\left[1-\sqrt{\frac{2}{3}} F(100 \mid 00)\right] .
$$

\section{Results and Discussion}

\subsection{Preliminaries}

As discussed in $\S 1$, the aim of this work is to derive transport properties required as input into a fluid model for streamer discharges. We consider the reduced electric field $E / n_{0}$ range: $0.01-1000 \mathrm{Td}\left(1 \mathrm{Td}=10^{-21} \mathrm{~V} \mathrm{~m}^{2}\right)$. A neutral temperature of $298 \mathrm{~K}$ is assumed and superelastic collisions are not allowed for. The cross sections for the electron scattering in $\mathrm{N}_{2}$ detailed by Stojanović and Petrović, ${ }^{21)}$ and cross sections for electron scattering in $\mathrm{O}_{2}$ developed by Itikawa et al. ${ }^{22,23)}$ are implemented in this work. For elastic collision processes we use the original Boltzmann collision operator while for non-elastic conservative processes we employ the Wang-Chang et al. ${ }^{14)}$ generalization. Attachment and ionization collision operators are detailed in ref. 15. We assume that in the division of post-collision energy between the scattered and ejected electrons in an ionization

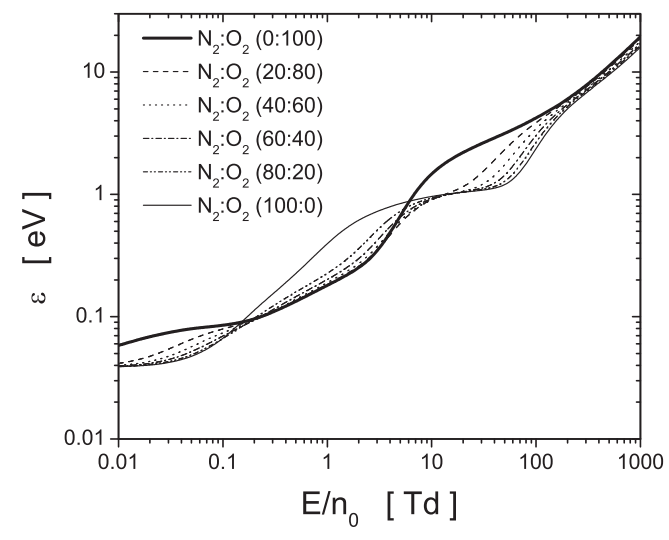

Fig. 1. Variation of the mean energy with $E / n_{0}$ for various $\mathrm{N}_{2}-\mathrm{O}_{2}$ mixtures.

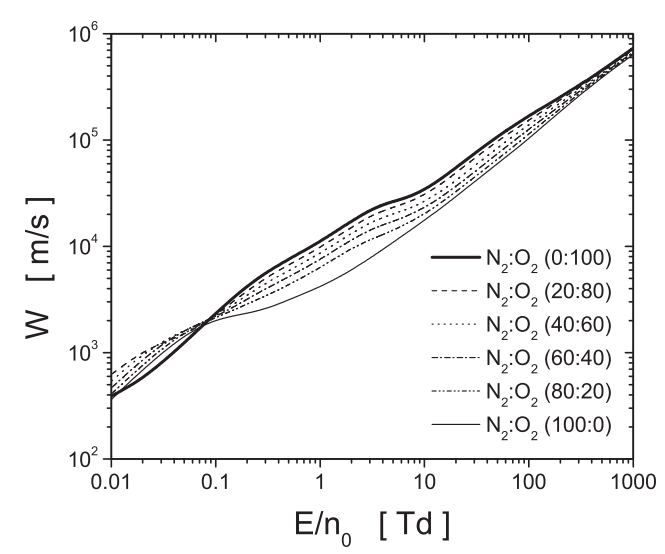

Fig. 2. Variation of the flux drift velocity with $E / n_{0}$ for various $\mathrm{N}_{2}-\mathrm{O}_{2}$ mixtures.

process, all fractions are equiprobable. At the high energies considered in this work, assumptions on the division of energy may considerably influence transport profiles. ${ }^{15,24)}$

\subsection{Electron transport coefficients in $\mathrm{N}_{2}-\mathrm{O}_{2}$ mixtures}

In Figs. 1 and 2 we display the variation of the mean energy and flux drift velocity, respectively, with the reduced electric field $E / n_{0}$ for various $\mathrm{N}_{2}-\mathrm{O}_{2}$ mixtures. The properties of the cross sections are reflected in the profiles of the mean energy. We see that the mean energy, as expected, is greatly affected by the addition of $\mathrm{O}_{2}$ to $\mathrm{N}_{2}$. In the limit of high $E / n_{0}$, the mean energy of electrons is relatively insensitive to the gas composition. From the profiles of the drift velocity in Fig. 2 we see that there are no signs of a negative differential conductivity (NDC) effect, i.e., the drift velocity is a monotonically increasing function of $E / n_{0}$ for all mixtures. However, in the limit of low $E / n_{0}$, the drift velocity exhibits a non-linear behavior with respect to the abundance of $\mathrm{O}_{2}$ in the mixture. On the other hand, in the limit of high values of $E / n_{0}$, this transport quantity, like the mean energy, shows little sensitivity to the $\mathrm{O}_{2}$ concentration in the mixture. This is a clear sign that collision frequencies in this energy range are essentially independent of the $\mathrm{O}_{2}$ abundance in the mixture.

In Fig. 3 we show the percentage differences between the bulk and the flux components of the drift velocity as a 


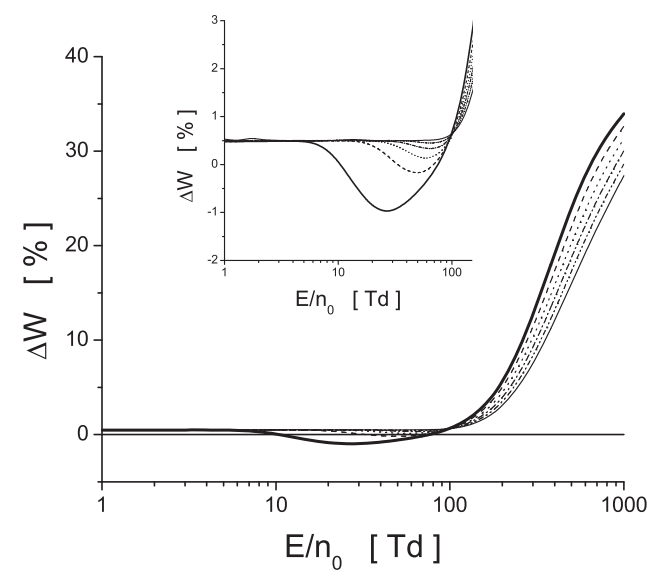

Fig. 3. The percentage difference between the bulk and flux components of the drift velocity as a function of $E / n_{0}$ for various $\mathrm{N}_{2}-\mathrm{O}_{2}$ mixtures.

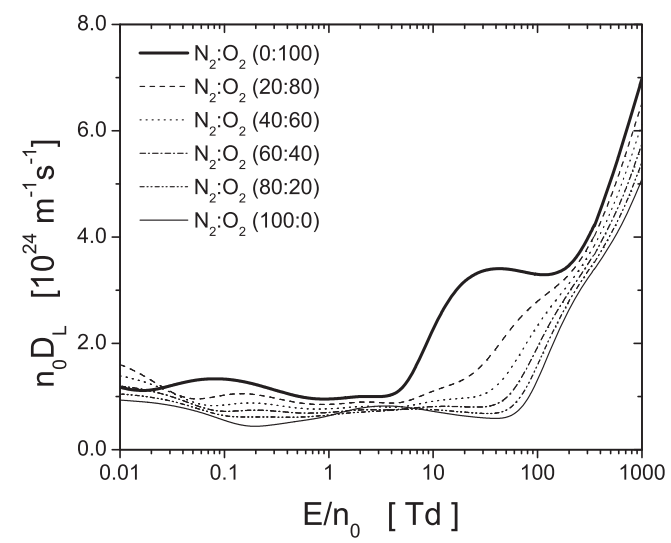

Fig. 5. Variation of the flux longitudinal diffusion coefficient with $E / n_{0}$ for various $\mathrm{N}_{2}-\mathrm{O}_{2}$ mixtures.

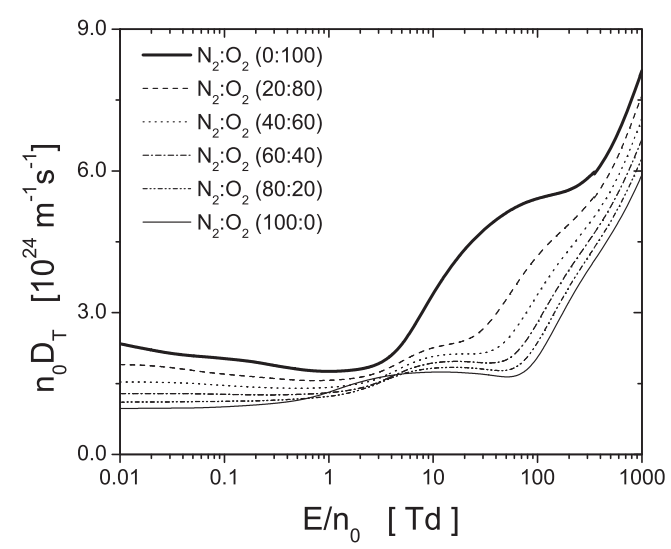

Fig. 6. Variation of the flux transverse diffusion coefficient with $E / n_{0}$ for various $\mathrm{N}_{2}-\mathrm{O}_{2}$ mixtures.

work, the diffusion becomes more isotropic in nature. The degree of anisotropy will have a bearing on anomalous longitudinal diffusion that may be observed in time varying fields. It is therefore of some concern that it is a common practice in streamer modeling to assume that diffusion is isotropic, i.e., $D=D_{\mathrm{L}}=D_{\mathrm{T}}$.

\subsection{The effects of three-body attachment}

In what follows we report on the influence of three-body attachment on the transport properties of electrons in pure oxygen. This process has been usually treated through the studies of two-body dissociative attachment that occurs for $E / n_{0}<10 \mathrm{Td}$, and as already emphasized only the limited number of theoretical investigations into the three-body, pressure dependent attachment process for electrons has been published. ${ }^{6-8)}$ In this section we briefly report how the three-body attachment in pure oxygen affects the transport data, the origin and mechanisms of transport phenomena induced by this process and possible physical implications which arise from its explicit inclusion into streamer macroscopic models.

It is now well known, that electron attachment to molecular $\mathrm{O}_{2}$ at low electron energies leading to the formation of stable $\mathrm{O}_{2}{ }^{-}$ion, is a three-body process which 


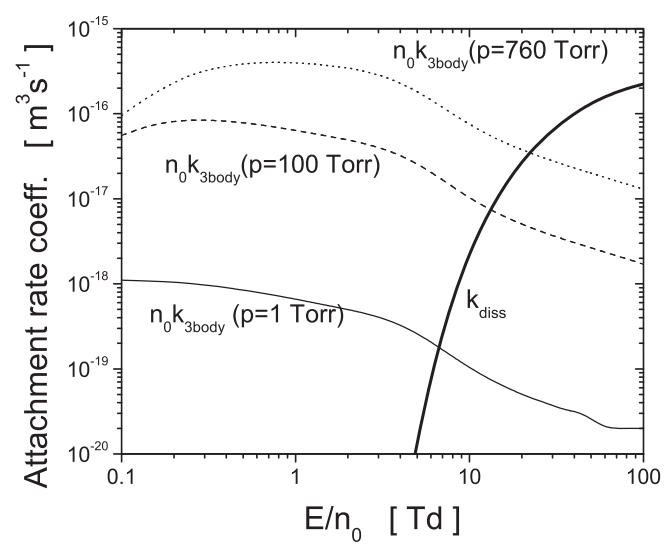

Fig. 7. Variation of the dissociative and three-body attachment rate coefficients with $E / n_{0}$ and pressures for electrons in pure $\mathrm{O}_{2}$.

proceeds in two stages. In the first stage, the electron is captured by the molecule into a vibrationally excited temporary negative ion-state, sometimes called a resonant state $\left(\mathrm{O}_{2}+e \rightarrow \mathrm{O}_{2}{ }^{-*}\right)$. This excited molecular ion may subsequently interact through auto-detachment to give a free electron and a neutral molecule which may be left in a vibrationally excited state $\left[\mathrm{O}_{2}^{-*} \rightarrow \mathrm{O}_{2}(\mathrm{v})+e\right]$. Alternatively, the excess energy of the molecular ion may be removed in a collision with a third body, which in the case of pure $\mathrm{O}_{2}$ is another $\mathrm{O}_{2}$ molecule $\left[\mathrm{O}_{2}{ }^{-*}+\mathrm{O}_{2} \rightarrow\right.$ $\mathrm{O}_{2}{ }^{-}$(stable $)+\mathrm{O}_{2}$ ]. As a result of this process we have one stable negative ion and neutral molecule whose internal state is characterized through a different set of quantum numbers imposed by energy conservation. It is clear that for a low concentration of $\mathrm{O}_{2}$ molecules the rate of attachment is controlled by the stabilizing collisions between vibrationally excited negative ions and neutral $\mathrm{O}_{2}$ molecules. Therefore, this process is a pressure-dependent process and it would be important to understand how this pressure-dependent non-conservative process manifests itself in the transport properties.

In Fig. 7 we show the three-body and dissociative attachment rate coefficients as a function of $E / n_{0}$ for three different pressures, as indicated on the graph. We see that the three-body attachment rate coefficient increases with pressure in the entire range of $E / n_{0}$. At the same time, as expected, the rate coefficient for dissociative attachment is a pressure-independent quantity. As the gas pressure increases, the attachment cross section becomes larger and the effect of electron loss on the distribution function in the energy region between thermal electron energies and approximately $1.5 \mathrm{eV}$, increases. As $E / n_{0}$ increases, however, the mean energy also increases and there are fewer electrons available for attachment. Hence, the effect of attachment on the electron distribution decreases, and for high enough $E / n_{0}$, this effect is insignificant and so the total attachment rate becomes pressure independent and solely determined by the dissociative attachment process (see Fig. 8).

In Fig. 9 we show the quantity $k_{3 \text { body }}$ as a function of $E / n_{0}$ for three different pressures. This figure shows that the threebody attachment rate coefficient decreases with pressure in the limit of low $E / n_{0}$, while as expected, for high $E / n_{0}$

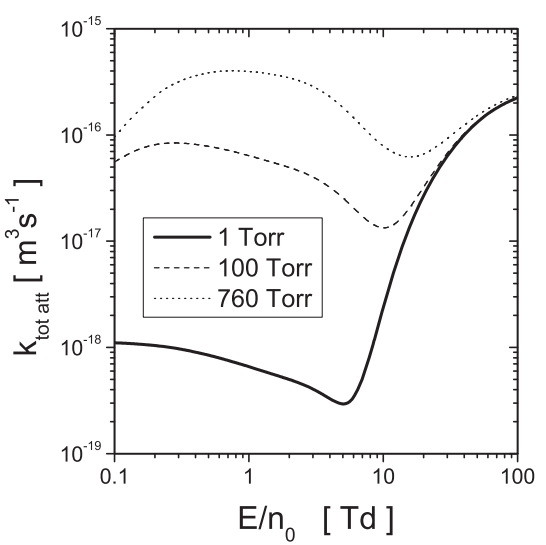

Fig. 8. Variation of the total attachment rate coefficient with $E / n_{0}$ and pressures for electrons in pure $\mathrm{O}_{2}$.

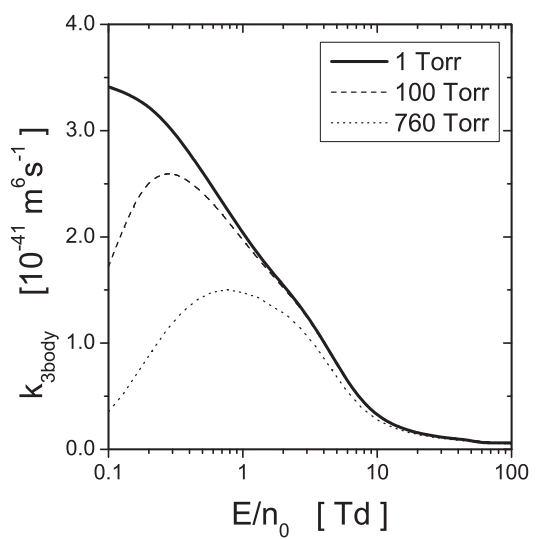

Fig. 9. Variation of the three-body attachment rate coefficient with $E / n_{0}$ and pressures for electrons in pure $\mathrm{O}_{2}$.

range this quantity becomes pressure-independent. It is clear that if $E / n_{0}$ increases (for a fixed pressure), then the mean energy also increases and the number of electrons that can be consumed by the attachment processes is significantly reduced. However, under conditions of varying pressure and fixed $E / n_{0}$ the situation is not so simple. It is clear that for an increasing pressure the cross section for three-body attachment also increases. If the inverse of the lifetime of auto-detachment significantly dominates the frequency of stabilizing collisions, then the cross section for attachment increases almost linearly with pressure and consequently there are no variations of the distribution function. Since the distribution function remains unchanged with the pressure, $k_{3 \text { body }}$ is a function of $E / n_{0}$ only and exhibits typical hydrodynamic behavior. Conversely, if the inverse of the lifetime for auto-detachment becomes comparable to the frequency for stabilizing collision of the three-body process, then the cross section for attachment does not increase linearly with pressure and the effect of electron loss on the distribution function increases. These effects induce pressure-dependence of the distribution function and hence $k_{3 \text { body }}$ is no longer a function of $E / n_{0}$ only. Under these non-hydrodynamic conditions, $k_{3 \text { body }}$ becomes pressuredependent quantity. Similar observations have been made by Taniguchi et al. ${ }^{6,7)}$ 


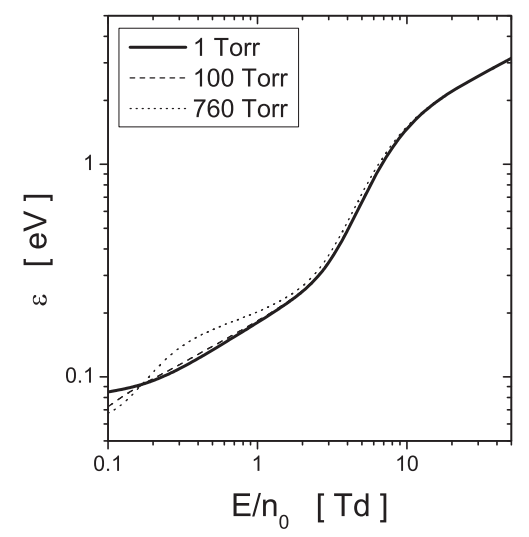

Fig. 10. Variation of the mean energy with $E / n_{0}$ and pressure for electrons in pure $\mathrm{O}_{2}$.

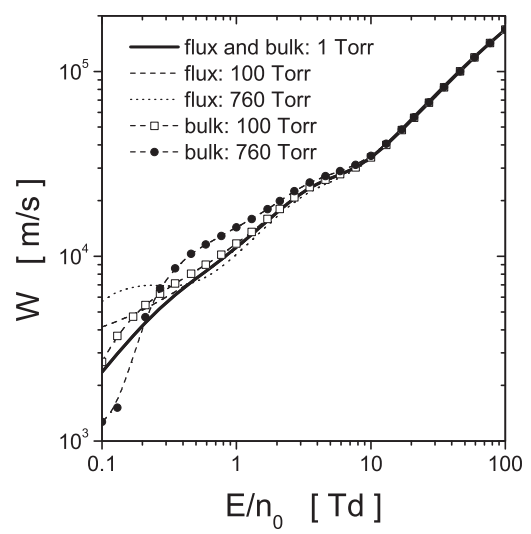

Fig. 11. Variation of the bulk and flux drift velocity components with $E / n_{0}$ and pressure for electrons in pure $\mathrm{O}_{2}$.

The pressure dependence of the transport coefficients arises through the pressure dependence of the attachment cross section. Figures 10 and 11 display the variation of the mean energy and drift velocity with $E / n_{0}$ and with pressure for electrons in pure oxygen. From the profiles of the mean energy we clearly see the regions of attachment cooling and attachment heating. Similar effects can be observed in the profiles of the bulk and flux components of the drift velocity. First we see that the flux is greater than the bulk which is a clear indication of the attachment cooling and then an opposite situation occurs: the bulk is greater than the flux which is a clear sign of the attachment heating. For low values of $E / n_{0}$ only the high-energy electrons in the swarm can undergo attachment. Thus attachment cooling occurs, and since the higher the pressure, the greater the attachment, we observe the mean energy to be less for the higher pressure. Also since attachment predominantly occurs at the front of the swarm this will cause a backward shift of the swarm's centre of mass and then a reduction of the measurable or bulk drift velocity component follows. However, since the swarm mean energy increases with $E / n_{0}$, we clearly see a point reached where the lower-energy electrons are preferentially lost due to the attachment. Attachment heating occurs and mean energy increases with pressure. At the same time, this phenomenon results in a forward shift of the swarm's centre of mass and then an increase in the measurable drift velocity follows. Other transport properties including the rate coefficients, gradient energy parameter and components of the temperature tensor are available from the authors on request.

\section{Conclusions}

In this work, we have presented the results of a systematic investigation of non-conservative electron transport in the mixtures of molecular nitrogen and oxygen under the influence of the electric field. We have considered conditions consistent with the electrons in a streamer discharge with partial motivation being the provision of transport coefficients to be employed in fluid modeling of such a discharge. These coefficients are accurate to within $1 \%$. In addition, we have examined the explicit and implict effects of threebody attachment on electron transport. It is found that threebody attachment induces a non-hydrodynamic behavior in the profiles of certain transport properties in the limit of thermal electron energy, e.g., the transport coefficients are pressure-dependent quantities.

\section{Acknowledgments}

SD and UE acknowledge support from STW-project 10118, part of the Netherlands' Organization for Scientific Research (NWO). SD and ZLjP are supported by the MNTR, Serbia, under the contract number ON171037. SD and RDW have also been supported by the Australian Research Council and the Centre for Antimatter-Matter studies, Australia.

1) U. Ebert, C. Montijn, T. M. P. Briels, W. Hundsdorfer, B. Meulenbroek, A. Rocco, and E. M. van Veldhuizen: Plasma Source Sci. Technol. 15 (2006) S118.

2) U. Ebert and D. Sentman: J. Phys. D 41 (2008) 230301.

3) L. R. Grabowski, E. M. van Veldhuizen, A. J. M. Pemen, and W. R. Rutgers: Plasma Chem. Plasma Process. 26 (2006) 3.

4) S. Babayan, J. Jeong, V. Tu, J. Park, G. Selwyn, and R. Hicks: Plasma Source Sci. Technol. 7 (1998) 286.

5) C. Li, U. Ebert, and W. Hundsdorfer: J. Phys. D 42 (2009) 202003.

6) T. Taniguchi, H. Tagashira, I. Okada, and Y. Sakai: J. Phys. D 11 (1978) 2281.

7) T. Taniguchi, K. Kawamura, S. Sakamoto, and H. Tagashira: J. Phys. D 15 (1982) 1187.

8) D. Spence and G. J. Schulz: Phys. Rev. A 5 (1972) 724.

9) N. Liu and V. P. Pasko: J. Geophys. Res. 109 (2004) A04301.

10) O. Eichwald, O. Ducasse, D. Dubois, A. Abahazem, N. Merbahi, M. Benhenni, and M. Yousfi: J. Phys. D 41 (2008) 234002.

11) M. M. Nudnova and A. Yu. Starikovskii: J. Phys. D 41 (2008) 234003.

12) A. Luque, V. Ratushnaya, and U. Ebert: J. Phys. D 41 (2008) 234005.

13) L. Boltzmann: Wein. Ber. 66 (1872) 275 [in German].

14) C. S. Wang-Chang, G. E. Uhlenbeck, and J. De Boer: in Studies in Statistical Mechanics, ed. J. De Boer and G. E. Uhlenbeck (Wiley, New York, 1964) Vol. II, p. 241.

15) K. F. Ness and R. E. Robson: Phys. Rev. A 34 (1986) 2185.

16) R. E. Robson and K. F. Ness: Phys. Rev. A 33 (1986) 2068.

17) K. F. Ness: Phys. Rev. E 47 (1993) 327.

18) S. Dujko, R. D. White, Z. Lj. Petrović, and R. E. Robson: Phys. Rev. E 81 (2010) 046403.

19) R. D. White, R. E. Robson, S. Dujko, P. Nicoletopoulos, and B. Li: J. Phys. D 42 (2009) 194001.

20) Z. Lj. Petrović, S. Dujko, D. Marić, G. Malović, Ž. Nikitović, O. Šašić, J. Jovanović, V. Stojanović, and M. Radmilović-Radjenović: J. Phys. D 42 (2009) 194002.

21) V. D. Stojanović and Z. Lj. Petrović: J. Phys. D 31 (1998) 834.

22) Y. Itikawa, A. Ichimura, K. Onda, K. Sakimoto, K. Takayanagi, Y. Hatano, M. Hayashi, H. Nishimura, and S. Tsurubuchi: J. Phys. Chem. Ref. Data 18 (1989) 23.

23) Y. Itikawa: J. Phys. Chem. Ref. Data 38 (2009) 1.

24) K. F. Ness and A. M. Nolan: Aust. J. Phys. 53 (2000) 437. 